\title{
Abundance of Veillonella spp. does not Reflect Salivary Nitrite Production after Nitrate Ingestion
}

\author{
Takahiro Mitsui ${ }^{1 *}$, Taichi Ishikawa ${ }^{2}$, Ryô Harasawa ${ }^{3}$, and Minoru Sasaki \\ ${ }^{1}$ Department of Home Economics, Faculty of Education, Iwate University, Morioka, Japan \\ ${ }^{2}$ Division of Molecular Microbiology, Department of Microbiology, Iwate Medical University, Yahaba, Japan \\ ${ }^{3}$ The Iwate Research Center for Wildlife Diseases, Morioka, Japan
}

Received: May 1, 2020 / Revised: May 20, 2020 / Accepted: June 1, 2020

\begin{abstract}
Veillonella spp. have been reported to be the most prevalent nitrate-reducing bacterial species in the oral cavity. The purpose of this study was to examine the relationship between the abundance of Veillonella spp. and nitrite production after nitrate ingestion. Bacterial samples were obtained from the tongue surfaces of 50 university students. The predominant Veillonella spp., V. atypica, $V$. dispar, and $V$. rogosae were identified and enumerated using real-time polymerase chain reaction (qPCR). Salivary nitrate and nitrite were measured before and 30,60 , and $90 \mathrm{~min}$ after ingestion of $100 \mathrm{ml}$ of beetroot juice. Increased nitrite concentrations were observed in all participants, with a mean increase of $0.61(0.42-1.10) \mathrm{mM}$ expressed as the median (interquartile range). Veillonella atypica was detected in 40 subjects (80\%), V. dispar in 48 $(96 \%)$, and $V$. rogosae in $48(96 \%)$, at quantities ranging from $1.3 \times 10^{2}$ to $2.8 \times 10^{7} \mathrm{CFU} / \mathrm{ml}$ per subject. The strengths of the correlations of the log colony forming unit (CFU) values of $V$. atypica, V. dispar, V. rogosae, and the $\log \mathrm{CFU}$ value of the three species together with the increase in nitrite levels were $0.091,0.114$, -0.228 , and 0.060 , respectively, none of which were significant $(p>0.05)$. Our results indicate that the abundance of Veillonella spp. is not related to salivary nitrite production after nitrate ingestion.
\end{abstract}

Keywords: Saliva, nitrate, nitrite, Veillonella, real-time polymerase reaction (qPCR)

\section{Introduction}

Dietary nitrate, which humans ingest mainly in leafy green vegetables such as spinach and lettuce, is absorbed rapidly into the bloodstream from the upper intestine [1]. Approximately $75 \%$ of circulating nitrate is excreted by the kidneys, and the rest may be distributed and stored throughout the body. A significant amount is reported to be concentrated in the salivary glands by an active transport system and then secreted into the oral cavity, where the nitrate concentration is thus up to 1020 times higher than that in plasma [2]. This oral nitrate is partially converted to nitrite by microbial

\section{*Corresponding author}

Tel: +81-19-621-6649, Fax: +81-19-621-6600

E-mail: mitsui@iwate-u.ac.jp

(c) 2020, The Korean Society for Microbiology and Biotechnology nitrate reductase activity, mainly on the surface of the tongue, which supports a high bacterial density [3]. The changes in salivary nitrate/nitrite that occur after nitrate ingestion vary markedly from individual to individual [4-6].

Salivary nitrite is further reduced to nitric oxide (NO) and other nitrogen oxides in the oral cavity and the upper intestine [7]. This nitrate-nitrite-NO pathway may have a vasodilatory effect, as endogenous NO is derived from L-arginine. The potential beneficial effects of dietary nitrate include reduced blood pressure [8, 9], reduced oxygen consumption [5], antimicrobial activity in the oral cavity [10,11], and upper intestinal tracts [12].

The human oral cavity houses more than 300 different facultative bacteria with effective nitrate reductase, though only some of these have been specifically impli- 
cated in the reduction of nitrate to nitrite [13]. Doel et al. [3] isolated and identified the major nitrate-reducing bacterial species on the tongues of healthy adults using 16S rDNA sequencing and found that Veillonella atypica (34\%), Veillonella dispar (24\%), Actinomyces odontolyticus (21\%), and Rothia mucilaginosa (10\%) were the most abundant. Using 16S rRNA metagenome sequencing, Hyde et al. [14] also identified Veillonella as the most abundant nitrate-reducing genus, together with Prevotella, Neisseria, and Haemophilus.

Veillonella spp. are a strictly anaerobic group, commonly seen on the tongue, with typical quantities ranging from $6.0 \times 10^{5}$ to $1.7 \times 10^{8} \mathrm{CFU} / \mathrm{ml}[15]$ and occupying from $3.0 \%$ to $36.3 \%$ [16] of the dorsal anaerobic colony count. So far, seven Veillonella species have been isolated from human oral cavities; among these, V. atypica, $V$. dispar, and $V$. rogosae are predominant [15-17].

According to previous studies, Veillonella spp. may produce a significant portion of salivary nitrite, and the variation in the quantity of the genus present in each individual's mouth is a possible cause of the inter-individual differences in salivary nitrite production after nitrate ingestion. As far as we know, there have been no previous studies investigating the relationship between the abundance of Veillonella spp. and salivary nitrite production level after nitrate loading. The purpose of this study is to detect and quantify the most common Veillonella spp. in the oral cavity and to compare their abundance to the level of salivary nitrite produced after nitrate ingestion.

\section{Methods}

\section{Participants and study protocol}

The experiment enrolled 52 (31 male and 21 female) non-smoking healthy university students with a mean age of $20.1 \pm 1.4$ (SD) years. None of the participants had used antibiotics or mouthwash in the last 3 months. Written informed consent was obtained from all participants, and the study protocol was approved by the Human Ethics Committee of Iwate University (No. 201407). Additionally, the study was conducted in accordance with the Declaration of Helsinki (version 2013).

All participants were asked to refrain from eating vegetables at dinner before the sampling day and to skip breakfast. They arrived at the laboratory prior to
7:30 a.m. and rested for $10 \mathrm{~min}$ before the experiment began. Just before the nitrate load was consumed, a 1.0$\mathrm{ml}$ whole saliva and bacteria sample was collected in the following manner: Each participant spat their saliva into a petri dish and the dorsal surface of their tongue was swabbed five times using a sterile cotton-wool stick to collect bacteria. Swabs are widely used to collect tongue biofilms in many studies including those on Veillonella spp. [15, 16]. To obtain sufficient samples for the PCR and qPCR procedures described below, this procedure was repeated three times. The stick was immediately placed in $1 \mathrm{ml}$ of phosphate-buffered saline (PBS) solution to create a bacterial suspension. Two bacterial suspensions were centrifugated at 13,000 rpm for $2 \mathrm{~min}$, and the supernatant was discarded for qPCR analysis.

Just after baseline collection, participants consumed $100 \mathrm{ml}$ of beetroot juice (James White Drinks, Ltd., $\mathrm{UK}$ ), which is equivalent to $372 \mathrm{mg}$ of nitrate. Afterwards, saliva samples were collected at 30-min intervals for $90 \mathrm{~min}$. Bacterial and saliva samples were stored at $-20^{\circ} \mathrm{C}$ immediately after collection.

\section{Analysis of salivary nitrate and nitrite}

The details of the analysis of salivary nitrate/nitrite were previously described [11]. Nitrite was determined colorimetrically by diazotization with sulphanilamide coupled with N-(1-naphthyl)ethylendiamine to form an azo-dye, and the samples were tested spectroscopically at $540 \mathrm{~nm}$. Nitrate was measured by the same method after being reduced to nitrite though a cadmium-copper column.

\section{Bacterial strain, culture conditions, and specific primers}

V. atypica NCTC 11830, V. dispar NCTC 11831, and $V$. rogosae JCM 15642 strains were cultivated overnight in brain heart infusion broth adjusted to a final concentration of $1 \%(\mathrm{w} / \mathrm{w})$ sodium lactate. The quality and purity of the bacterial culture were checked by Gram staining. The overnight broth culture was diluted with PBS to an $\mathrm{OD}_{600}$ of 0.1 .

At the same time, the number of bacterial cells of each strain was counted by plating $100 \mu \mathrm{l}$ of $10^{-3}, 10^{-4}$, and $10^{-5}$ dilutions onto Columbia agar with $5 \%$ sheep's blood (BD, Tokyo, Japan) and then incubating for 7 days at $37^{\circ} \mathrm{C}$ in a container with Anaeropack-Anero (Mitsubishi Gas Chemical, Japan). The following species-specific forward 
Table 1. Primers used for qPCR assays.

\begin{tabular}{|c|c|c|c|c|}
\hline Target species & Sequences (5' 3') & $\operatorname{Tm}\left({ }^{\circ} \mathrm{C}\right)$ & Product size (bp) & Reference \\
\hline \multirow[t]{2}{*}{ V. atypica } & F:TCTCTTTGGGAAGAATTAGAACGC & 82.7 & 384 & {$[18]$} \\
\hline & R:GTGTAACAAGGGAGTACGGACC & & & \\
\hline \multirow[t]{2}{*}{ V. dispar } & F:GTGTAACAAGGGAGTACGGACC & 82.0 & 321 & {$[18]$} \\
\hline & R:GTGTAACAAGGGAGTACGGACC & & & \\
\hline \multirow[t]{2}{*}{ V. rogosae } & F:TGCAGAAGATGTAACAGTAAGC & 79.9 & 215 & [18] \\
\hline & R:GGATGCTCCAGAAATGGATC & & & \\
\hline
\end{tabular}

The primers for $V$. rogosae were modified versions of the original sequences.

primers in the $r p o B$ gene were used for identification: ATYF (5'-TCTCTTTGGGAAGAATTAGAACGC-3') for V. atypica and DISF (5'-AACGCGTTGAAATTCGTCATGAAC-3') for $V$. dispar. The reverse primer VR (5'-GTGTAACAAGGGAGTACGGACC-3') is commonly used for these two species [18]. Because the PCR products made using ROGF (5'-ATTGCAGAAGATGTAACAGTAAGC3') for $V$. rogosae and VR are overweight for qPCR, 604 bp [18], the modified versions of these primers 5'TGCAGAAGATGTAACAGTAAGC-3' and 5'-GGATGCTCCAGAAATGGATC-3', respectively, were used. The expected sizes of the PCR products were $384 \mathrm{bp}$ for $V$. atypica, $321 \mathrm{bp}$ for $V$. dispar, and $215 \mathrm{bp}$ for $V$. rogosae. The primers were synthesized by Bex Co., Ltd. (Tokyo, Japan). The primers used in this study are summarized in Table 1.

To confirm the DNA band of each primer, PCR agarose gel procedures were performed for all participants. The details of these procedures have been previously described [6].

\section{DNA extraction and preparation of qPCR standard curve}

A 1-ml sample of each bacterial $\mathrm{OD}_{600}$ of 0.1 suspension and its dilutions (i.e., $10^{-1}, 10^{-2}, 10^{-3}, 10^{-4}$, and $10^{-5}$ ) with PBS were centrifugated at 13,000 rpm for $2 \mathrm{~min}$ and the supernatant was discarded. Then, $40 \mu \mathrm{l}$ of $0.05 \mathrm{M} \mathrm{NaOH}$ was added and the resulting mixture was heated at $90^{\circ} \mathrm{C}$ for $10 \mathrm{~min}$. After cooling, $6.4 \mu \mathrm{l}$ of $1 \mathrm{M}$ Tris- $\mathrm{HCl}(\mathrm{pH} 7.5)$ was added and the mixture was centrifugated at 15,000 rpm for $2 \mathrm{~min}$ [19]. The supernatant was used to create a standard curve for qPCR to quantify the target bacteria. Each reaction mixture $(25 \mu \mathrm{l})$ contained $1 \mu \mathrm{l}$ of DNA sample, $12.5 \mu \mathrm{l}$ of SYBR Premix Ex Taq II (2×), $0.5 \mu \mathrm{l}$ of forward and reverse primer $(50 \mathrm{pmol} / \mu \mathrm{l})$, and $10.5 \mu \mathrm{l}$ of TEA buffer. Thermal cycling was performed and consisted of $95^{\circ} \mathrm{C}$ for $30 \mathrm{~s}, 35$ cycles of $95^{\circ} \mathrm{C}$ for $5 \mathrm{~s}$, and $60^{\circ} \mathrm{C}$ for $30 \mathrm{~s}$ with the Thermal Cycler Dice real-time PCR system II (Takara Bio Inc., Japan). The cycle threshold $(\mathrm{Ct}$ ) was defined as the cycle number at which a sample's fluorescence increment crossed the threshold. The $\mathrm{Ct}$ was used to quantify the number of bacteria compared against the standard curve.

The sample bacterial suspensions from the tongue biofilm were treated in the same manner. Before qPCR amplification, extracted DNA concentrations were evaluated at $260 \mathrm{~nm}$ using a NanoDrop 2000 spectrophotometer (Thermo Fisher Science, USA).

\section{Data analysis}

Data are expressed as mean \pm SD or median (interquartile range) when the data are abnormally distributed as assessed using the Shapiro-Wilk test. Undetectable quantities (ND) are regarded as zero. The increases in concentration of salivary nitrate ( $\Delta$ nitrate) and nitrite ( $\Delta$ nitrite) from the baseline level to the highest value at 30,60 , or 90 min were obtained. The correlation coefficient between $\Delta$ nitrate and $\Delta$ nitrite, the log colonyforming unit (CFU) values for each Veillonella species and for all three species together, and $\Delta$ nitrite were calculated. Statistical significance was set at $p<0.05$. All statistical analyses were performed using Excel 2016 for Windows with add-in soft BellCurve for Excel (Social Survey Research Information Co., Ltd., Japan).

\section{Results}

One male participant and one female participant were excluded from the data analysis because the quantity of extracted DNA was not sufficient (i.e., $<2 \mathrm{ng} / \mu \mathrm{l}$ ) for qPCR analysis. 
Table 2. Salivary nitrate/nitrite concentrations after beet juice ingestion.

\begin{tabular}{cccccc}
\hline & Fasting & $30 \mathrm{~min}$ & $60 \mathrm{~min}$ & $90 \mathrm{~min}$ & $\Delta$ increased level \\
\hline Nitrate & $0.25(0.14-0.44)$ & $1.91(0.97-2.75)$ & $1.81(1.12-3.09)$ & $1.84(1.23-2.83)$ & $1.94(1.30-3.65)$ \\
Nitrite & $0.26(0.18-0.35)$ & $0.77(0.55-1.11)$ & $0.78(0.53-1.19)$ & $0.73(0.53-1.13)$ & $0.61(0.42-1.10)$ \\
\hline
\end{tabular}

Data are expressed as median (interquatile range), $\mathrm{mM}$.

The concentrations of nitrate/nitrite during the sampling period are shown in Table 2 . The baseline nitrate/ nitrite concentrations were $0.25(0.14-0.44) \mathrm{mM}$ and $0.26(0.18-0.35) \mathrm{mM}$, respectively. Increased nitrate and nitrite concentrations after nitrate loading were seen in all participants, although inter-individual variation was large: 1.94 (1.30-3.65) $\mathrm{mM}$ for nitrate and $0.61(0.42-$ 1.10) $\mathrm{mM}$ for nitrite.

The $\mathrm{OD}_{600}$ values of 0.1 suspensions were $160 \times 10^{6}$ $\mathrm{CFU} / \mathrm{ml}$ for $V$. atypica, $7.7 \times 10^{6} \mathrm{CFU} / \mathrm{ml}$ for $V$. dispar, and $10 \times 10^{6} \mathrm{CFU} / \mathrm{ml}$ for $V$. rogosae. The standard curves were $\mathrm{Y}=-3.324 \mathrm{X}+43.742$ for $V$. atypica, $\mathrm{Y}=-3.318 \mathrm{X}+$ 36.910 for $V$. dispar, and $\mathrm{Y}=-3.327 \mathrm{X}+36.696$ for $V$. rogosae (Fig. 1). The squared correlation coefficients were 1.000 for all species. The detection limits of Ct were 32.15 for $V$. atypica, 29.75 for $V$. dispar, and 28.64 for $V$. rogosae, which were equivalent to $3056 \mathrm{CFU} / \mathrm{ml}, 145$ $\mathrm{CFU} / \mathrm{ml}$, and $242 \mathrm{CFU} / \mathrm{ml}$, respectively.

$V$. atypica, $V$. dispar, and $V$. rogosae were detected in 40 (80\%), 48 (96\%), and 48 (96\%) participants. At least one species was detected in all participants, but the total quantity of all species varied considerably among indi- viduals, ranging from $1.3 \times 10^{2}$ to $2.8 \times 10^{7} \mathrm{CFU} / \mathrm{ml}$. The $\log$ number values of $V$. atypica and $V$. dispar were strongly correlated $\left(\mathrm{r}^{2}=0.795, p<0.001\right)$, while the relationships between other species were weak, $\mathrm{r}=0.296$ $(p<0.05)$ for $V$. atypica-V. rogosae and $0.125(p>0.05)$ for $V$. dispar-V. rogosae (Fig. 2).

An agarose gel electrophoresis image is shown in Fig. 3. A DNA band for the PCR products of $V$. dispar was detected in all participants, while a band for $V$. atypica was detected in seven (14\%) and a band for $V$. rogosae in nine (18\%).

The relationships between the log curves of $V$. atypica, $V$. dispar, $V$. rogosae and all species together and $\Delta$ nitrite were $0.091,0.114,-0.228$, and 0.060 , respectively, none of which were significant (Fig. 4).

\section{Discussion}

To our knowledge, this is the first study to detect and quantify the main oral Veillonella spp. using qPCR. The extremely high sensitivity of this technique allowed us to detect each species with an accuracy of $\geq 80 \%$. Our
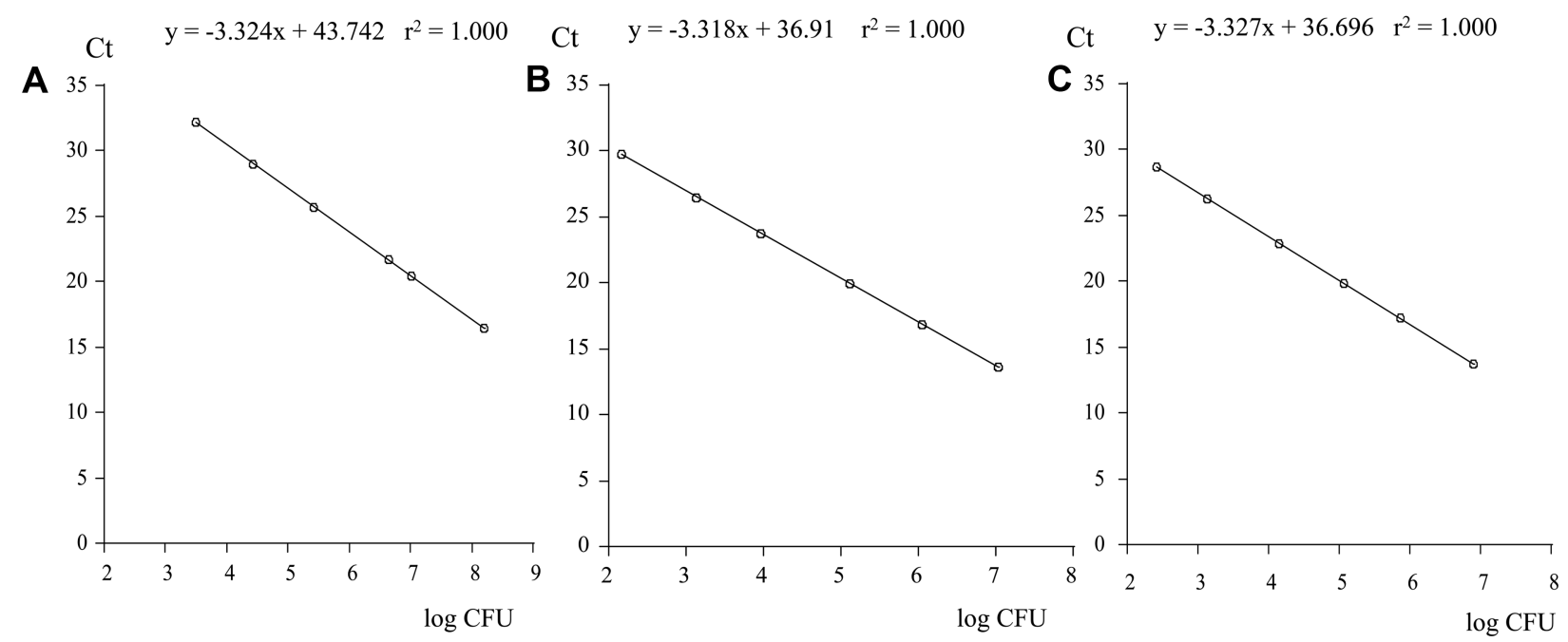

Fig. 1. Standard curves obtained from serial dilutions of DNA extracted from a culture in brain heart infusion broth; (A) $V$. atypica, (B) V. dispar, and (C) V. rogosae. 

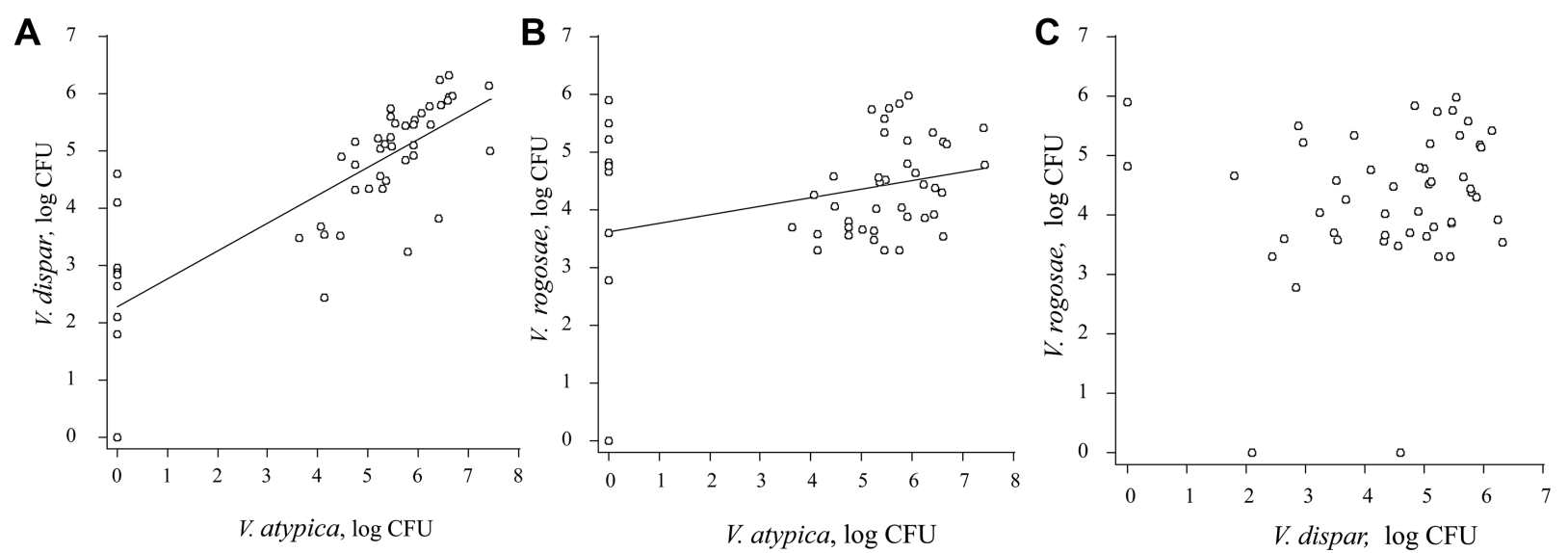

Fig. 2. The associations between log numbers of the species under study; $(A) r^{2}=0.795, p<0.001,(B) r^{2}=0.296, p<0.05$, and $(C) r^{2}=0.125, p>0.05$.

results ranged from $1.3 \times 10^{2}$ to $4.1 \times 10^{6} \mathrm{CFU} / \mathrm{ml}$. The ability to quantify bacterial loads in $\mathrm{CFU} / \mathrm{ml}$, which incorporates both $\mathrm{Ct}$ value and colony count, is another merit of qPCR. Although multiple species are implicated in nitrite production, investigating multiple factors (as in a metagenome analysis) is not appropriate in every study. The data describe a species' relative abundance (i.e., the proportion of the community that consists of that genus/species) as opposed to a concrete value. In addition, the sensitivity of $16 \mathrm{~S}$ rRNA metagenome anal$y$ sis is inferior to that of qPCR targeting the rpo $B$ gene adopted in this study. In particular, 16S rRNA analysis does not reliably differentiate the Veillonella species [16, $18,20]$.
The baseline levels of nitrate/nitrite, mostly derived from endogenous formation and recent dietary intake, were relatively low and stable. On the other hand, the $\Delta$ nitrate and $\Delta$ nitrite after nitrate loading varied significantly from individual to individual. Boss et al. [4] observed large variation between subjects in salivary nitrate/nitrite concentrations after nitrate loading, and concluded that it was possible to discriminate between subjects with consistently high or low nitrite conversion rates. We recently reported that the correlation coefficients between two measurements of $\Delta$ nitrate and $\Delta$ nitrite after nitrate ingestion in the same individual, separated by a 7-day interval, were 0.637 and 0.583 , respectively $(\mathrm{n}=24, p<0.01)$ [6]. The inter-individual

\section{V. atypica V. dispar V.rogosae V. atypica V. dispar V. rogosae}
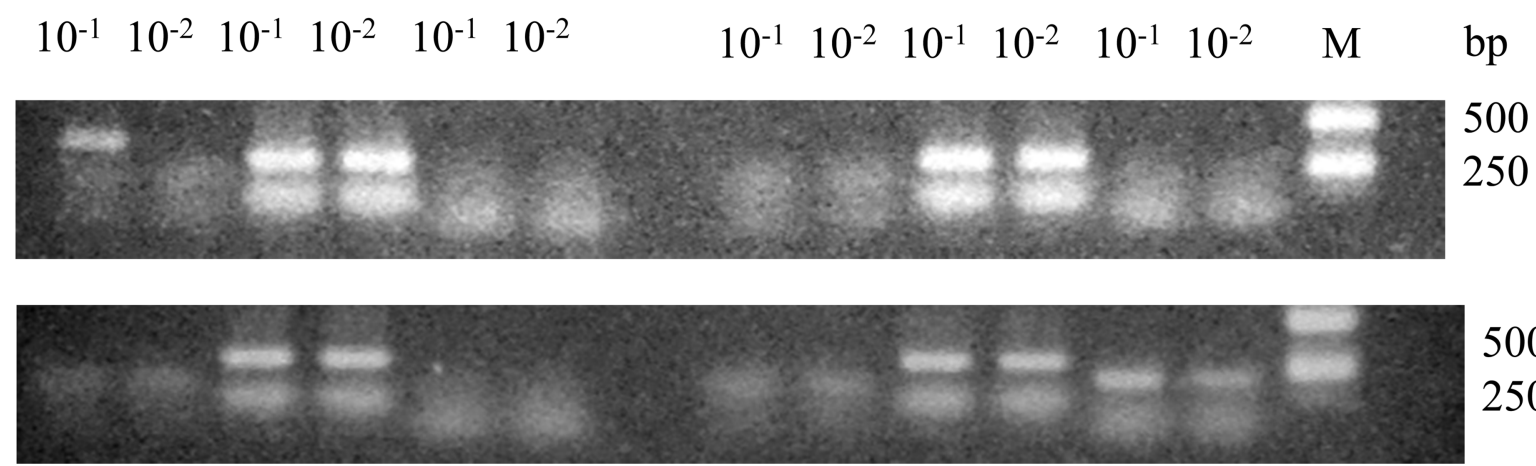

Fig. 3. Analysis of direct PCR products of V. atypica, V. dispar, and V. rogosae from saliva diluted with $10^{-1}$ and $10^{-2}$. The brightness of the photographs of electrophoretograms was adjusted using Image J (NIH, https://imagej.nih.gov/ij/). 

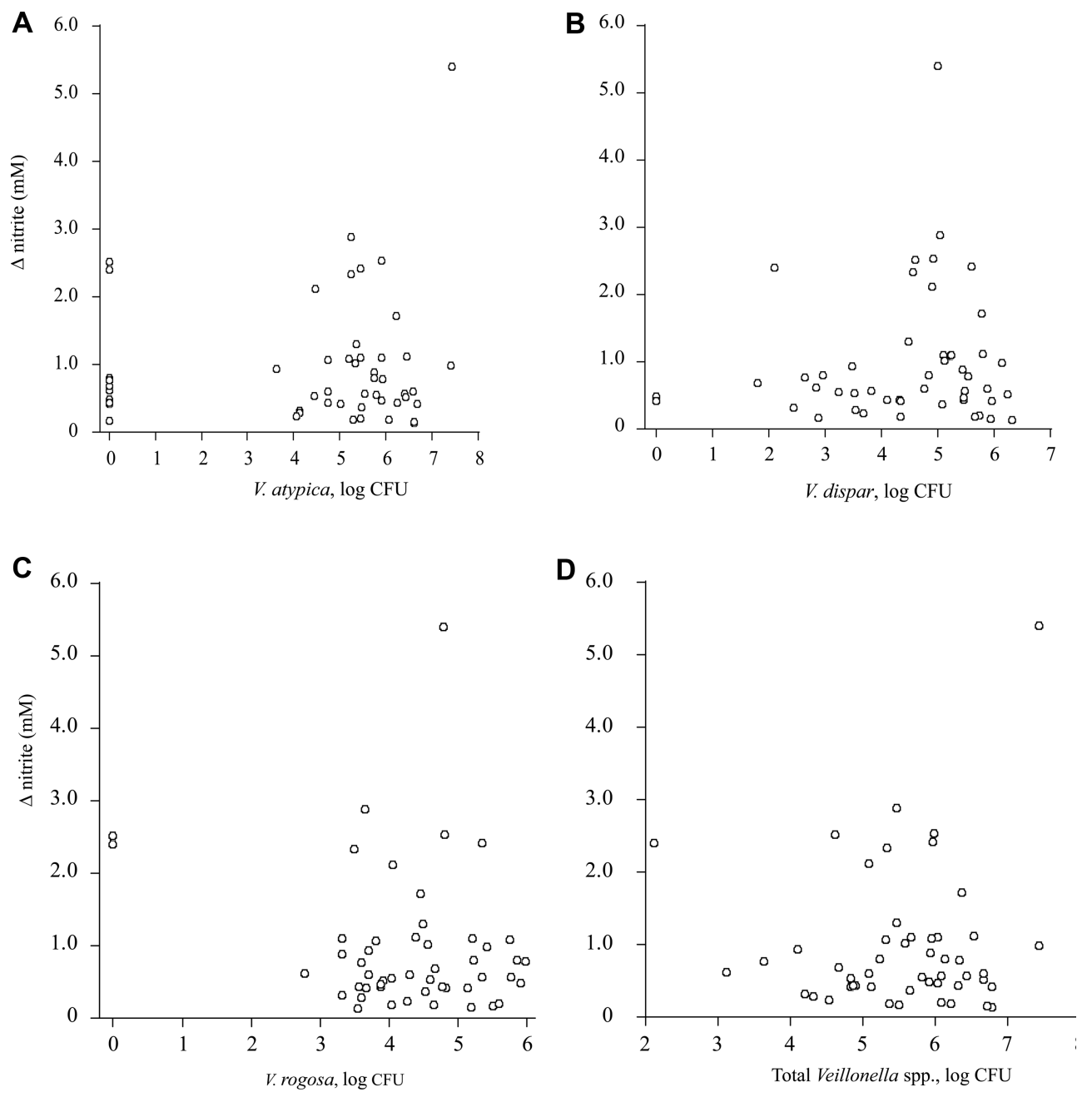

Fig. 4. The relationship between salivary nitrite production after nitrate load and CFU/ml of each or sum of Veillonella spp; (A) $r^{2}=0.091, p>0.05$, (B) $r^{2}=0.014, p>0.05$, (C) $r^{2}=-0.228, p>0.05$, and (D) $r^{2}=0.0597, p>0.05$.

differences in $\Delta$ nitrate may result from variations in certain physiological abilities such as absorption in the upper intestine, excretion by the kidney, concentration by the salivary gland, and salivary flow rate [13]. On the other hand, the nitrate-nitrite-NO pathway is a nonmammalian metabolic process that can be completely abolished by the use of chlorhexidine-based mouthwash, although salivary nitrate is little affected by such treatment [21].

In contrast to our hypothesis, we observed a very weak association between the abundance of any or all of the Veillonella spp. and nitrite production (Fig. 4). If Veillonella spp. makes a major contribution to nitrate reduction in the oral cavity as suggested by Doel et al. [3], we would expect to obtain a moderate correlation coefficient $(r=0.40-0.59)$ [22]. The number of participants in this study (50) seems sufficient given that the total sample size required to determine whether a correlation coefficient, $\mathrm{r}=0.40-0.59$, differs from zero is $20-47$ when $\alpha=$ 0.05 and power is set to 0.80 . Our results, in which $\mathrm{r}=$ 0.059 and $n=50$, indicate that the relationship between the number of Veillonella and nitrite production is very weak.

Significant salivary nitrite production in those with lower Veillonella abundance is probably due to other nitrate-reducing species. On the other hand, relatively small nitrite production in participants with higher Veillonella abundance is not clear. Because the amounts of metabolites strongly reflect the numbers of bacteria [23], we predicted significant nitrite production would be 
observed in those with a higher abundance of Veillonella spp. One possible explanation for this is the relatively low nitrate reduction ability of these species. Doel et al. [3] concluded that Veillonella spp. were the most prevalent taxa and accordingly predicted that they would make a major contribution to nitrate reduction in the oral cavity. In fact, however, they found that the nitrate-reducing ability of $V$. atypica and $V$. dispar is much weaker than that of other spices such as Actinomyces odontolyticus and Rothia mucilaginosa in vitro. Similar results were observed by Hyde et al. [14], who reported a higher abundance of Veillonella spp. but a lower nitrate-reducing ability compared with Actinomyces.

Recently, Burleigh et al. [24] observed that those with a higher relative abundance of nitrate-reducing bacteria (> 50\%) produced more salivary nitrite after beet juice ingestion than did those with a lower relative abundance (<50\%) using 16S rRNA metagenome analysis. They found $V$. dispar to be the second most predominant species with relative abundance, $20.55 \pm 12.31 \%$ (SD), behind only Prevotella melaninogenica, $42.12 \pm 10.09 \%$. Probably because of the discrepancy of analytical methods, 16S rRNA metagenome vs. rpo $B$ qPCR, our results are partially inconsistent with their findings. However, higher (relative) abundance of a nitrate-reducing gene in a sample does not always mean that these taxa are also active or that they generate higher nitrite production.

Direct PCR is a particularly effective method for processing low-yield samples without culturing and DNA extraction [25] although its sensitivity seems to be inferior to that of qPCR. We detected $V$. dispar in all subjects, $V$. atypica in seven (14\%), and $V$. rogosae in nine (18\%). The discrepancy between the incidence rates measured by the two methods is not clear; the fact that the $\mathrm{Ct}$ values for $V$. atypica were approximately three times greater than those for $V$. dispar may indicate the lower specificity of the specific primer. The PCR enzyme and the primer employed in this study may also have a high specificity for $V$. dispar, which resulted in a $100 \%$ detection ratio.

Although it is beyond the scope of this study, the strong association between the CFU numbers of $V$. atypica and $V$. dispar ( $\mathrm{r}=0.795$, Fig. 3A) not having been seen in any other pair of species is interesting. The cause is not entirely unknown, as there may be a symbiotic relationship between these species. This result may be in keeping with the previous studies by Mashima et al. [15] that identified two groups of oral Veillonella spp. based on their distributions and frequencies. $V$. rogosa was identified as the predominant species in one group, while the other group consisted mainly of $V$. atypica and $V$. dispar.

One limitation that may have affected our results is that the measurements were taken only once for each individual. Salivary nitrate/nitrite production and tongue bacteria may change over time as they are continuously affected by various factors such as diet, water consumption, teeth cleaning, flakes of epithelium, and secretions. Intra-individual differences should also be carefully considered.

In conclusion, we detected Veillonella spp. in the oral cavity in all participants at concentrations on the order of $10^{2}$ to $10^{7} \mathrm{CFU} / \mathrm{ml}$ using qPCR. The relationship between $\log$ CFU/ml of Veillonella spp. and the increase in nitrite concentration after nitrate loading was not statistically significant in spite of the prevalence of these species. We conclude that the abundance of Veillonella spp. is not related to salivary nitrite production after nitrate ingestion.

\section{Acknowledgments}

This work was supported by a Grant from the Organization for Research Promotion, Iwate University. The authors would like to thank Dr. Yu Shimoyama for designing the primer for V. rogosae.

\section{Conflict of Interest}

The authors have no financial conflicts of interest to declare.

\section{References}

1. McKnight GM, Duncan CW, Leifert C, Golden MH. 1999. Dietary nitrate in man: friend or foe? Br. J. Nutr. 81: 349-358.

2. Eisenbrand G, Spiegelhalder B, Preussmann R. 1980. Nitrate and nitrite in saliva. Oncology 37: 227-231.

3. Doel JJ, Benjamin N, Hector MP, Rogers M, Allaker RP. 2005. Evaluation of bacterial nitrate reduction in the human oral cavity. Eur. J. Oral Sci. 113: 14-19.

4. Bos PM, Van den Brandt PA, Wedel M, Ockhuizen T. 1988. The reproducibility of the conversion of nitrate to nitrite in human saliva after a nitrate load. Food Chem. Toxicol. 26: 93-97.

5. Larsen FJ, Schiffer TA, Ekblom B, Mattsson MP, Checa A, Wheelock $C E$, et al. 2014. Dietary nitrate reduces resting metabolic rate: a 
randomized, crossover study in humans. Am. J. Clin. Nutr. 99: 843850.

6. Mitsui T, Saito M, Harasawa R. 2018. Salivary nitrate-nitrite conversion capacity after nitrate ingestion and incidence of Veillonella spp. in elderly individuals. J. Oral Sci. 60: 405-410.

7. Duncan C, Dougall $H$, Johnston $P$, Green S, Brogan R, Leifert C, et al. 1995. Chemical generation of nitric oxide in the mouth from the enterosalivary circulation of dietary nitrate. Nat. Med. 1: 546551.

8. Jonvik KL, Nyakayiru J, Pinckaers PJ, Senden JM, van Loon LJ, Verdijk LB. 2016. Nitrate-rich vegetables increase plasma nitrate and nitrite concentrations and lower blood pressure in healthy adults. J. Nutr. 146: 986-993.

9. Sobko T, Marcus C, Govoni M, Kamiya S. 2010. Dietary nitrate in Japanese traditional foods lowers diastolic blood pressure in healthy volunteers. Nitric Oxide 22: 136-140.

10. Doel JJ, Hector MP, Amirtham CV, Al-Anzan LA, Benjamin N, Allaker RP. 2004. Protective effect of salivary nitrate and microbial nitrate reductase activity against caries. Eur. J. Oral Sci. 112: 424428.

11. Mitsui T, Fujihara M, Harasawa R. 2013. Salivary nitrate and nitrite may have antimicrobial effects on Desulfovibrio species. Biosci. Biotechnol. Biochem. 77: 2489-2491.

12. Dykhuizen RS, Fraser A, McKenzie $H$, Golden M, Leifert C, Benjamin N. 1998. Helicobacter pylori is killed by nitrite under acidic conditions. Gut 42: 334-337.

13. Hezel MP, Weitzberg E. 2015. The oral microbiome and nitric oxide homoeostasis. Oral Dis. 21: 7-16.

14. Hyde ER, Andrade F, Vaksman Z, Parthasarathy K, Jiang H, Parthasarathy DK, et al. 2014. Metagenomic analysis of nitratereducing bacteria in the oral cavity: implications for nitric oxide homeostasis. PLoS One 9: e88645.

15. Mashima I, Kamaguchi A, Nakazawa F. 2011. The distribution and frequency of oral Veillonella spp. in the tongue biofilm of healthy young adults. Curr. Microbiol. 63: 403-407.
16. Beighton D, Clark D, Hanakuka B, Gilbert S, Do T. 2008. The predominant cultivable Veillonella spp. of the tongue of healthy adults identified using rpoB sequencing. Oral Microbiol. Immunol. 23: 344-347.

17. Mashima I, Liao YC, Miyakawa H, Theodorea CF, Thawboon B, Thaweboon S, et al. 2018. Veillonella infantium sp. nov., an anaerobic, Gram-stain-negative coccus isolated from tongue biofilm of a Thai child. Int. J. Syst. Evol. Microbiol. 68: 1101-1106.

18. Igarashi E, Kamaguchi A, Fujita M, Miyakawa H, Nakazawa F. 2009. Identification of oral species of the genus Veillonella by polymerase chain reaction. Oral Microbiol. Immunol. 24: 310-313.

19. Rudbeck L, Dissing J. 1998. Rapid, simple alkaline extraction of human genomic DNA from whole blood, buccal epithelial cells, semen and forensic stains for PCR. Biotechniques 25: 588-590.

20. Marchandin $H$, Teyssier $C$, Siméon De Buochberg $M$, Jean-Pierre $\mathrm{H}$, Carriere $\mathrm{C}$, et al. 2003. Intra-chromosomal heterogeneity between the four $16 \mathrm{~S}$ rRNA gene copies in the genus Veillonella: implications for phylogeny and taxonomy. Microbiology 149: 1493-1501.

21. Govoni M, Jansson EA, Weitzberg E, Lundberg JO. 2008. The increase in plasma nitrite after a dietary nitrate load is markedly attenuated by an antibacterial mouthwash. Nitric Oxide 19: 333337.

22. Evans JD. 1996. Straightforward statistics for the behavioral sciences. Pacific Grove, CA: Brooks/Cole Publishing.

23. Roszak DB, Colwell RR. 1987. Metabolic activity of bacterial cells enumerated by direct viable count. Appl. Environ. Microbiol. 53: 2889-2893.

24. Burleigh MC, Liddle L, Monaghan C, Muggeridge DJ, Sculthorpe N, Butcher JP, et al. 2018. Salivary nitrite production is elevated in individuals with a higher abundance of oral nitrate-reducing bacteria. Free Radic. Biol. Med. 120: 80-88.

25. Cavanaugh SE, Bathrick AS. 2018. Direct PCR amplification of forensic touch and other challenging DNA samples: A review. Forensic Sci. Int. Genet. 32: 40-49. 